\title{
Translation Studies in the Frame of Blending
}

\author{
Chunmei Tong \\ Henan Institute of Engineering, Zhengzhou, China
}

\begin{abstract}
The thesis focuses on the structure and its translation in the frame of conceptual integration, which suggests that the analysis of the ST means de-integrate the schematic structures; translation process is a bi-lateral integration of the ST and TT, both syntactically and schematically.
\end{abstract}

Keywords-translation; integration; ST; TT

\section{INTRODUCTION}

In this thesis, the researcher discusses some issues pertaining to translation studies in the frame of blending. First of all, the researcher puts forward conceptual blending point of view towards translation, which suggests that the analysis of the ST means de-integrate the schematic structures, then, the translator integrates the schematic structures with corresponding syntactic patterns in the TT. Translation process is a bi-lateral integration of the ST and TT, both syntactically and schematically. According to the relations between the source and target languages in terms of communicative mode, translation processes can be classified into four types of integration networks: simplex and mirror networks, as well as single-scope and double-scope networks.

\section{Perspectives ON TRANSLATION StUdies}

Translation has been practiced for thousands of years, and debates on the nature of translation have been part of translation practiced for almost as long. However, translation theories develop rather slowly. The growth of translation studies as a separate discipline only took shape in the late of 20th century. Thus, the subject has developed in many parts of the world and is clearly continuing developing in 21st century. Translation studies bring together work in a wide variety of fields, including linguistics, literary study, history, anthropology, psychology and economics.

Until now, translation studies have undergone a long period of time and also amazing achievements have been made in this area. Whereas, few researchers have gone deep into the internal mechanism that manipulate the processes of translation and interpretation, both of whom just focus their attention on the surface factors, such as social and cultural factors, linguistic factors and so on. Only few translation theories discuss translation as a cognitive process, (rather than as a linguistic product). For instance, Jacobson (1966:23) defines translation as "the interpretation of verbal signs by means of another language”. Sager (1993:122) noted in his regard that the term interpretation itself incorporates "a whole series of cognitive processes which occur in translators' mind”. Some translation theorists just pay attention to the strategies that are applied to the process of translation. Neubert (1991:25) defines translation in terms of a series of problem-solving processes: problem identification, comprehension, retrieval, monitoring, problem reduction, and decision-making.

What surprises most is that very little research exists on the interface between cognitive aspects of the translation process, that is, the interface between linguistic transfer operations and its mental representation. Thus far, the literature on translation has dealt principally with evaluation and perspectives issues on how translations should be done. (Larson, 1984). In the following years, the view of translation as linguistic science has led to the creation of formal models of the translation process. For instance, the translation theory developed by Nida and Taber (1969), is based on concepts from transformational grammar, which can not disclose the real essence of translation and obviously can not achieve a satisfactory TT.

The analysis of translation examples in the thesis, which have already mentioned in the introduction, will focus on the cognitive operations involved in the process of translation that based on the blending theory that will be introduced in the next section. Translation, as Larson (1984:3) notes, is basically a process of changing of forms, that is, the translation process consists of changing a text in one language into a text in another language. The text "form" is the actual words, phrases, clauses, sentences, paragraphs, etc., which compose the text, and when translators look at translation examples, what translators see are forms of the source and the target languages. However, from a view of conceptual blending, these forms are the results of dynamic conceptual and linguistic blending operations. Now the questions have been raised: What is conceptual integration? How does blending operate in the process of communication and translation? These questions will be answered in the next sections of the thesis.

\section{TRANSLATION STUDIES IN THE FRAME OF BLENDING}

\section{A. Translation is the Result of Bilateral Integration of the ST and TT}

In the perspective of blending, translation is the result of the bi-literal integration of the ST and TT. Translator receives the linguistic expressions of the ST as an input, which triggers the conceptual structures of the ST, then; the communicative mode in a culture in turn takes shape, by which schemata of the communicative event can be drawn [1]. Up to this point, the first stage of translation is accomplished, which can be considered as the reverse process of blending.

As to any kind of ST, it must be the schemata of the ST that take shape first, then, through the integration of the communicative mode in a culture and the semiotic system which concretely behaves all kinds of genres such as novel and poetry in macro level and concrete wording and 
sentencing in micro level, the TT finally can take shape. Simply speaking, a text is an integration of a certain kind of communicative mode in a culture with a certain kind of semiotic system. As to the first stage of translation, the degeneration is a reverse of the blending, as have mentioned before, with the chief mission of the translator at this stage can be considered as disclosing subjective imagination structures of the ST. In fact, de-integration is a rather opening process, which means different translators may obtain different schemata of the communicative events based on different experiential backgrounds of their own. Having obtained the schemata of the communicative events, the translator enters into the second stage of translation, during which translator aims at seeking the effective communicative mode in a culture It then, the translator integrates the mode with the semiotic system in the target language, thus the TT is accomplished [2]. Up to this point, the whole process of translation is completed.

Whereas, the re-integration is far from easy in the process of translation practice. It is how to choose the communicative mode in a culture in the target language that annoys translators most. Firstly, take the culture of the target language itself as consideration, translators have different expressive modes with one event; secondly, there always exist many choices of the communicative modes in the cultures between the source and target languages. Therefore this choice will be an everlasting opening process, which can give an explanation why a ST has so many different TTs. The relations between the source and target languages in terms of communicative mode can be:

(1) Totally the same, which means that the imaginative objects and the thus constructed image schemata in both source and target languages have the same pragmatic function in the process of translation;

(2) Partially the same, which means that two different imaginative objections in the source and target languages have the same pragmatic function in the process of translation;

(3) Totally different, which means that the same imaginative objection in the source and target languages has totally different pragmatic functions in the process of translation;

(4) Lacking, which means that one imaginative objection in the communication mode in a culture does not exist in another. Serious considerations are worth taking on how to adjust the lacking, which is also an opening process.

\section{B. Four Types of Integration Networks}

According to the blending theory, human cognitive activities are governed by a series of rules, which based on four types of integration networks: simplex networks, mirror networks, single-scope networks and double-scope networks [3:119-135]. Since translation can be considered as a kind of communication, translation processes also be classified into four types of integration. In the next section, four types of networks of translation will be introduced one by one.

Simplex networks and mirror networks belong to the mental mapping that is shaped in a same constructive frame. That is, all the four spaces INPUT1, INPUT2, the generic space and the blending have the same framing structure.
Simplex and mirror integration networks reflect the common cognitive structures of human beings, which cannot be disturbed by different communicative modes in different cultures. In the translation process, it means that the imaginative objects in the communicative mode and thus constructed schemata of the communicated events, together with their pragmatic functions are totally the same between the source and the target languages.

In the thesis, the translation of CM constructions belongs to this category. As will be argued in the next chapter, both English and Chinese share a common conceptual structure to express $\mathrm{CM}$ constructions, that is, A causes $\mathrm{B}$ move to $\mathrm{C}$, which expresses a simple event that takes place all around the world. Therefore, in the process of translation, cultural factors can be neglected and attention should be transferred to grammatical blending between the ST and TT [4]. The blending process will be discussed in the later studies.

Single-scope networks contain the blending that INPUT1 and INPUT2 have different constructive frames, which share some similarities and each can be interpreted with the other. The frame of the blending only comes from one of the input space, INPUT1 or INPUT2, which is selected by the translator. In the process of de-integration, the translator may reconstruct the schemata of the author with his own experiences as the constructive frames, thus the translator can disintegrate and reconstruct the ST just by his own understanding, or translator may refurbish his own encyclopedia knowledge with the author's schemata. As have mentioned above, due to the differences of translators' encyclopedia knowledge and the different styles of the reconstruction and refurbishment, the selective process is an everlasting changing and opening one. In the process of translation, the selection is between domestication and foreignization. If schemata of the communicative events of the source language are selected as the frames of the blending, the selection should be considered as foreignization; if schemata of the communicative events of the target language are selected as the frame of the blending, the selection should be considered as domestication. The translator can make a choice by the purpose of translation, which is a subjective choice by the agent. Generally speaking, it is the schemata of the communicative events and the thus exerting pragmatic functions between the source and target languages that trigger the emergence of single-scope networks in the process of translation.

Double-scope networks have most complex integration modes, of which the constructive frames of the blends come from both INPUT1 and INPUT2, both of which have rather complex constructive frames. For instance, INPUT1 and INPUT2 may share no analogical characteristics or even have upside-down causalities, while it is in this blending process that new ideas, conceptual structures and communicative modes are created. Therefore, it is still a selective problem on how and how much does the constructive frame of the blending come from that of INPUT1 and INPUT2. In the process of de-integration, the translator may face the problem that his experiences cannot map with that of the author's. Sometimes the translator in vain tries to refurbish the constructive frames of the ST, therefore he has to abandon both frames and create new ones. This kind of de-integration is 
creative in nature, for the schemata that created by the translator are new to both himself and the author of the ST. So, it is obviously that the created ones are neither created by foreignization nor domestication; they are totally new integrations [5: 269-277]. As single-scope and double-scope networks are irrelevant to the study of the thesis, discussion in detail will be omitted here.

\section{SUMMARY}

Conceptual integration (blending) provides a way of the mental processes of mappings between languages and thoughts. If a source text is treated as the blends of the source language structures and schemata of the communicative events, in translating, when translators digest the source text, translators are actually disintegrating the text, and disclose the schemata of the communicative events integrated by the ST, and when actually translating, translators integrate the digested schemata from the source text with the target language structures [6]. Translating is, as a matter of fact, mental processes of embodied minds; a way to illustrate how the same communicative event is mapped onto different language structures in different translators' mind. Cognitive models of conceptual integration offer penetrating insights in this regard.

In the frame of conceptual integration, the relations between source and target languages in terms of the communicative mode can be: totally the same; partially the same; totally different and lacking. Corresponding to the four relations translation processes can be classified as four types of integration networks: simplex networks, mirror networks, single-scope and double-scope networks. As to E-C translation of the CM constructions, translators should focus their attention on the simplex and mirror networks since both English and Chinese share the same communicative mode to express CM concept with no cultural divergences. In the following section, an elaborate study on CM constructions in English and Chinese in the frame of blending should be carried out, so as to lay a foundation for the contrastive and translation studies in the late chapters. In the first part of next chapter, introduction of a brief literature review on the studies of CM constructions is necessary.

\section{ACKNOWLEDGMENT}

The thesis is sponsored by $\mathrm{Ph}$. $\mathrm{D}$ funds of Henan University of Engineering (2014034).

\section{REFERENCES}

[1] Danks, J. Cognitive Processes in Translation and Interpretation. Thousand Oaks and Landon: Sage, 1997.

[2] Fauconnier, G. \& Turner, M. Blending as a central process of grammar. In Conceptual Structure, Discourse, and Language, Ed, Adele Doldburge. Stanford: Centre for the study of Language and Information. , 1996.

[3] Fauconnier, G. \& Turner, M. “Conceptual Integration Networks.” Cognitive Science 22.2: 133-187,1998.

[4] Fauconnier, G. \& Turner, M. Conceptual Projection and Middle Spaces [technical report]. UCSD. Available form http://hci.ucsd.edu/cogsci/tech.htm, 1994.

[5] Fauconnier, G. \& Turner, M. Principles of Conceptual Integration: Discourse and Cognition: Bridging the Gap. Stanford: Centre for the study of language and information. P: 269-284, 1998.
[6] Fauconnier, G. and Turner, M. Optimality Principles and Conceptual Integration. UCB/UCSD Workshop in cognitive linguistics, January, 1996. Available form http://www.wam.umd.edu/mturner/blending.html, 2006. 